\title{
Análisis Comparativo del Número de Neurofilamentos en Nervios Isquiáticos de Rata Sometidos a Neuropraxia Trata- das con Láser de Baja Intensidad y Ultrasonido Terapéutico
}

\author{
Comparative Analysis of the Number of Neurofilaments in Rat Sciatic Nerve \\ Undergoing Neuropraxia Treated by Low-Level Laser and Therapeutic Ultrasound
}

Matamala, F."; Cornejo, R."; Paredes, M."; Farfán, E.**; Garrido, O. S. ${ }^{* * * *}$ \& Alves, N.****

MATAMAla, F.; CORNEJO, R.; PAREDES, M.; FARFÁN, E.; GARRIDO, O. S. \& ALVES, N. Análisis comparativo del número de neurofilamentos en nervios isquiáticos de rata sometidos a neuropraxia tratadas con láser de baja intensidad y ultrasonido terapéutico. Int. J. Morphol., 32(1):369-374, 2014.

RESUMEN: Es habitual que tras una compresión nerviosa se aplique terapia, ya sea, a través de laser de baja intensidad (LBI) o ultrasonido (US). El objetivo de este trabajo fue determinar la efectividad de dichos tratamientos para reparar el citoesqueleto neuronal evaluando la variación en el número de neurofilamentos. Se realizó un diseño experimental, en el cual se utilizaron 30 ratas que fueron

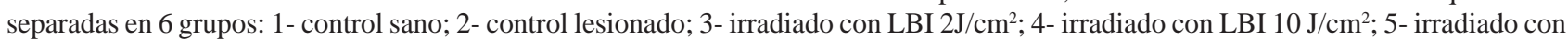
US $0,5 \mathrm{~W} / \mathrm{cm}^{2}$ y 6 - irradiado con US $1 \mathrm{~W} / \mathrm{cm}^{2}$. Con excepción del grupo 1 los especímenes fueron anestesiados y se les realizó la compresión del nervio isquiático derecho utilizando una presión de $40 \mathrm{~N}$ durante 45 segundos. Veinticuatro horas después de la compresión se inició la irradiación con LBI y US, según protocolo. En nuestra investigación constatamos que el incremento en el número de neurofilamentos se relacionó con la dosis aplicada de LBI y US. El valor medio de neurofilamentos/0,25 mm² obtenidos en cada grupo fue: 1 - 128; 2 - 100; 3 - 156; 4 - 140; 5 - 100; 6 - 148. La aplicación de LBI de y de US terapéutico aumenta el número de neurofilamentos en nervios isquiáticos de rata sometidos a neuropraxia, siendo el LBI más eficaz en comparación al US. Se agrega que estas terapias para inducir la regeneración del nervio lesionado se relacionan al tipo de protocolo utilizado, lo que demuestra la necesidad de establecer la adecuada dosis de irradiación con el propósito de obtener la mejor respuesta terapéutica.

PALABRAS CLAVE: Nervio isquiático; Compresión nerviosa; Neurofilamentos; Terapia por láser de baja intensidad; Terapia por ultrasonido.

\section{INTRODUCCIÓN}

La neuropatía periférica causada por una lesión es un problema clínico habitual. La gravedad o grado de la lesión del nervio periférico es uno de los determinantes más importantes para la recuperación del paciente. Clínicamente, entre las lesiones nerviosas compresivas la neuropraxia es la más leve, existiendo un bloqueo fisiológico de conducción nerviosa sin interrupción axonal. Morfológicamente se puede observar desmielinización segmentaria, sin degeneración Walleriana (Seddon \& Herbert, 1975), y desde el punto de vista clínico los pacientes pueden presentar afecciones motoras y sensitivas resultando en pérdida funcional importante y disminución de la calidad de vida (Jaquet et al., 2001).
Como parte del citoesqueleto neuronal se observan las neurofibrillas, dentro de las cuales los neurofilamentos (NF) son fundamentales para formar una estructura resistente, siendo abundantes en los axones, los que pueden estar constituidos casi totalmente por NF. Estos corresponden a filamentos intermedios formados por polímeros de tripletes proteicos de 3 subunidades de tubulina y con una masa molecular de 60KDa a 200KDa (Afifi \& Bergman, 2005). La cantidad de NF es proporcional al calibre de la fibra nerviosa (Hoffman et al., 1984), siendo la fibra nerviosa el principal determinante de la velocidad de conducción de impulsos a lo largo del axón (Hursh, 1939).

\footnotetext{
Departamento de Ciencias Básicas, Facultad de Medicina, Universidad de La Frontera, Temuco, Chile.

* Departamento de Anatomía, Escuela de Medicina, Pontificia Universidad Católica, Santiago, Chile.

**** Instituto de Ciencias Marinas y Limnológicas, Universidad Austral de Chile,Valdivia, Chile.

***** CIMA, Facultad de Odontología, Universidad de La Frontera, Temuco, Chile.

Fuente Financiamiento: Proyecto DI 12-0056. Dirección Investigación. Universidad de La Frontera.
} 
El ultrasonido (US) consiste en una terapia que entrega energía térmica en forma directa sobre los tejidos provocando un aumento en el metabolismo local y de la lesión, contribuyendo a los procesos reparativos (Martínez Morillo et al., 1998). Ebenbichler et al. (1998) han demostrado que el US puede mejorar los síntomas, así como la velocidad de conducción del nervio mediano en pacientes con neuropraxia.

Estudios en animales han comprobado que el US terapéutico aumenta la velocidad de conducción nerviosa tras lesión inducida por estrés (Lowdon et al., 1988) y la reparación del nervio después de su neurotomía (Crisci \& Ferreira, 2002).

En el campo médico la efectividad de la irradiación láser es cada vez más evidente. La terapia por laser de baja intensidad (LBI) produce un efecto bioestimulante, antiinflamatorio y analgésico a través de la irradiación directa sin provocar respuesta térmica (Snyder-Mackler \& Collender, 1995). Varios investigadores han estudiado el efecto terapéutico del LBI y han demostrado que la irradiación con LBI acelera la recuperación de la sensibilidad (Solé et al., 2012) y disminuye el tiempo de retorno de la función de pacientes con lesión de nervios periféricos (Miloro \& Repasky, 2000). Estudios en animales mostraron que la irradiación LBI mejora los procesos regenerativos de los nervios periféricos tras completa resección y anastomosis (Shamir et al., 2001) además de aumentar la tasa de crecimiento de los axones y la mielinización, acelerando y mejorando la regeneración de la lesión (Rochkind \& Ouknine, 1992).

El objetivo de este estudio fue determinar la efectividad de la terapia por LBI y del US terapéutico para reparar el citoesqueleto neuronal analizando la variación en el número de NF de los nervios isquiáticos de ratas Sprague Dawley sometidas a neuropraxia.

\section{MATERIAL Y MÉTODO}

Para este estudio fueron utilizadas 30 ratas de la cepa Sprague Dawley, peso promedio de $250 \mathrm{~g}$, las cuales fueron separadas en 6 grupos denominados: 1- Control sano; 2Control lesionado; 3- Lesionado sometido a irradiación láser (GaAs, $904 \mathrm{~nm}, 10 \mathrm{~mW}, 2 \mathrm{~J} / \mathrm{cm}^{2}$, 4 puntos, spot $0,1 \mathrm{~cm}^{2}$, $60 \mathrm{~s} /$ punto, 10 días consecutivos); 4- Lesionado sometido a irradiación láser (GaAs, $904 \mathrm{~nm}, 10 \mathrm{~mW}, 10 \mathrm{~J} / \mathrm{cm}^{2}, 4$ puntos, spot $0,1 \mathrm{~cm}^{2}, 60 \mathrm{~s} /$ punto, 10 días consecutivos); 5- Lesionado sometido a irradiación con US (3,2 MHz, ERA 1,9 $\mathrm{cm}^{2}, 0,5 \mathrm{~W} / \mathrm{cm}^{2}, 60 \mathrm{~s}$, modo continuo, 10 días consecutivos); 6- Lesionado sometido a irradiación con US (3,2 MHz, ERA $1,9 \mathrm{~cm}^{2}, 1,0 \mathrm{~W} / \mathrm{cm}^{2}, 60 \mathrm{~s}$, modo continuo, 10 días consecutivos).
Con excepción del grupo 1 todos los especímenes fueron anestesiados con Ketamina $(0,5 \mathrm{ml})$ y Xilacina $(0,6$ $\mathrm{ml}$ ) y se aisló quirúrgicamente el nervio isquiático derecho, el cual fue comprimido con pinza ad hoc durante 45 segundos y con una presión de $40 \mathrm{~N}$. A las 24 h después de la compresión se inició la terapia con LBI (grupos 3 y 4) y con US (grupos 5 y 6). A los 28 días después de realizarse la neuropraxia los especímenes se eutanasiaron con sobredosis de anestésico y sus nervios fueron extraídos y preparados para microscopía electrónica de transmisión con un aumento de $14000 \mathrm{X}$.

Las muestras en estudio correspondieron a segmentos del cabo distal de nervios isquiáticos lesionados por compresión. Para cada uno de los nervios procesados se tomaron 2 fotografías de microscopía electrónica con 14000 X de aumento y se enumeraron las muestras del uno al seis, de acuerdo a cada grupo.

En cada microfotografía se contó el número de NF por área de superficie, utilizando la técnica que consiste en sobreponer una lámina transparente del tamaño de la microfotografía, dividida en cuadrados (Fig. 1), los cuales miden 0,7 x $0,7 \mathrm{~cm}\left(0,49 \mathrm{~cm}^{2}\right)$, y que de acuerdo a la escala indicada por el microscopio electrónico, cada cuadrado corresponde a 0,5 x 0,5 micras $\left(0,25 \mathrm{~mm}^{2}\right)$ (Fig.1).

Luego al sobreponerse la respectiva lámina a cada una de las microfotografías se realizó la medición; para mejorar la observación de estos elementos se utilizó una lupa convencional, que no modificó la escala de medición descrita anteriormente. En cada una de las fibras nerviosas se midieron zonas seleccionada en forma aleatoria, al interior de este cuadrado de $0,49 \mathrm{~cm}^{2}$ equivalente a $0,25 \mathrm{~mm}^{2}$ (Fig. 2A y B).

Una vez obtenidos los resultados se efectuó el análisis estadístico con el programa Stata 10.0 aplicando el test Shapiro Wilk.

\section{RESULTADOS}

La Tabla I muestra los valores mínimos, medios y máximos de los datos recolectados acerca del número de NF por área, de cada una de las muestras.

Se observó que los valores del grupo 3 (LBI 2 J/ $\mathrm{cm}^{2}$ ) superan al valor máximo del grupo 2 (control lesionado) y aun alcanzan valores superiores al grupo 1 (control sano) (Tabla I). Sobre el grupo 4 (LBI $10 \mathrm{~J} / \mathrm{cm}^{2}$ ) se constató que sus valores también superan al valor máximo del control lesionado y que se encuentran ubicados 


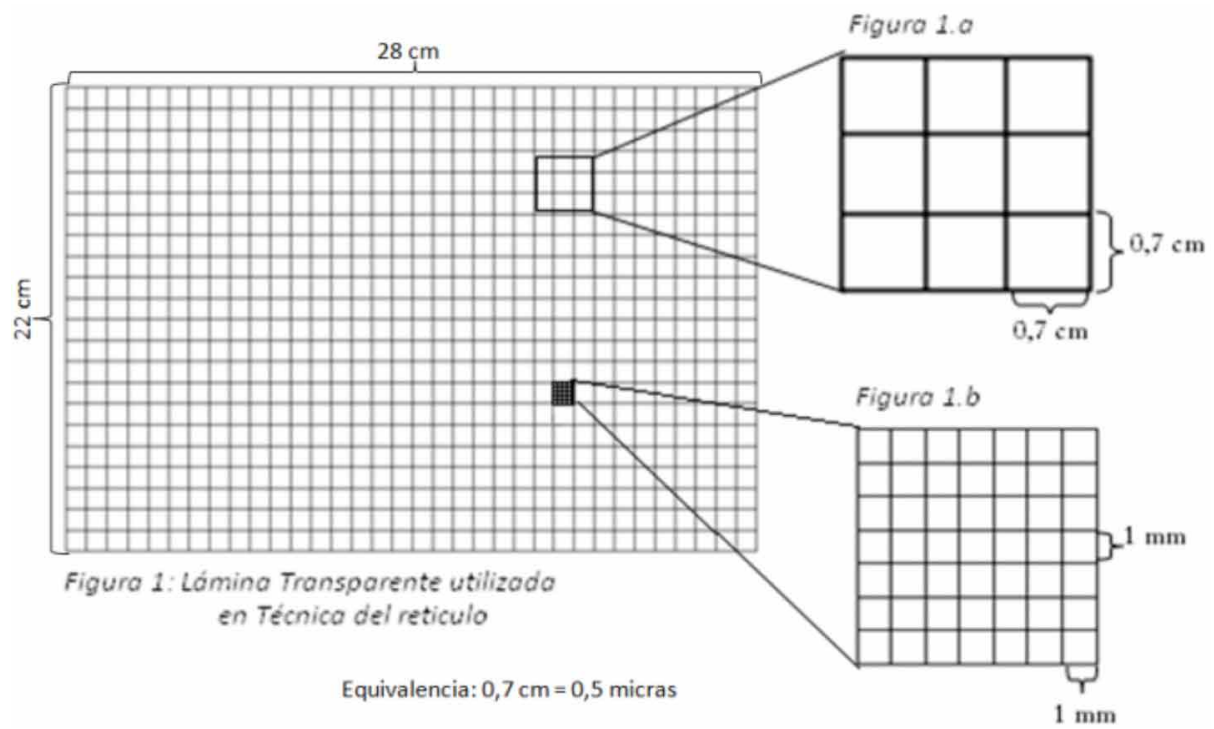

Fig. 1. Imágen que muestra la aplicación de la técnica utilizada.

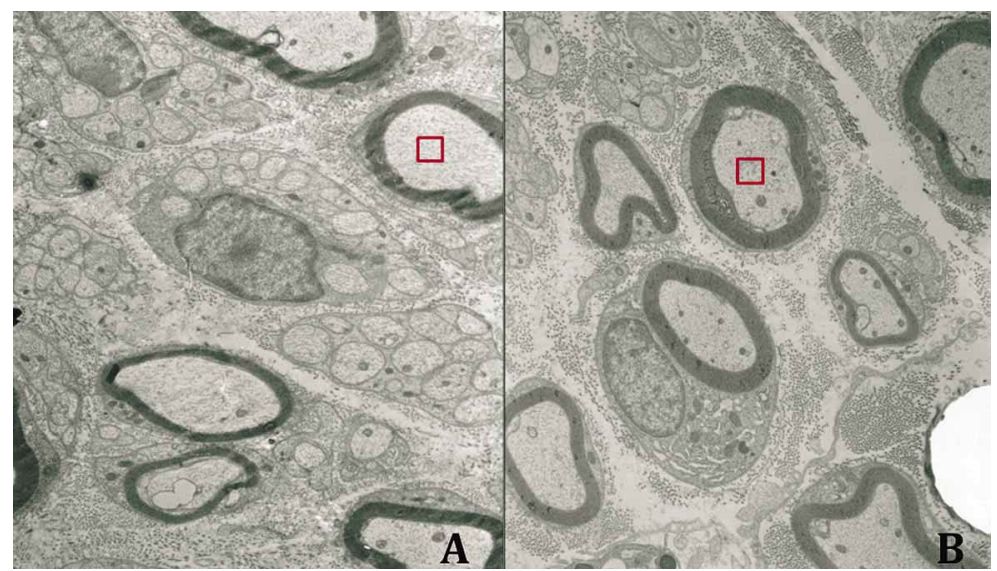

Fig. 2. Microfotografias de un corte transversal de nervio isquiático. A. Irradiado con US $1 \mathrm{~W} / \mathrm{cm}^{2} .14 .000 \mathrm{X}$; B. Irradiado com LBI $2 \mathrm{~J} / \mathrm{cm}^{2} .14 .000 \mathrm{X}$; ? Un área con neurofilamentos $14.000 \mathrm{X}$.

Tabla I. Valores mínimos, medios y máximos de neurofilamentos/0,25 mm².

\begin{tabular}{lccc}
\hline \multicolumn{1}{c}{ Grupos } & Mínimo & Medio & Máximo \\
\hline Grupo 1 (control sano) & 108 & 128 & 160 \\
Grupo 2 (control lesionado) & 88 & 100 & 124 \\
Grupo 3 (irradiación LBI 2 J/cm ${ }^{2}$ ) & 128 & 156 & 184 \\
Grupo 4 (irradiación LBI 10J/cm ${ }^{2}$ ) & 132 & 140 & 156 \\
Grupo 5 (irradiación US $0,5 \mathrm{~W} / \mathrm{cm}^{2}$ ) & 92 & 100 & 124 \\
Grupo 6 (irradiación US $1,0 \mathrm{~W} / \mathrm{cm}^{2}$ ) & 88 & 148 & 188 \\
\hline
\end{tabular}

entre el valor medio (128) y el valor máximo (160) del control sano (Tabla I). Sobre el grupo 5 (US $\left.0,5 \mathrm{~W} / \mathrm{cm}^{2}\right)$ constatamos que los valores se encuentran en un rango muy similar al control lesionado (Tabla I). En el grupo 6 (US $1 \mathrm{~W} / \mathrm{cm}^{2}$ ) observamos que el valor medio (148) y valor máximo (188) superan el valor medio (100) y va- lor máximo (124) del control lesionado.

Por lo tanto, se determinó que los valores medios de los grupos 3 , 4 y 6 superan los valores del control lesionado. Además se puede observar que los grupos 3 y 6 superan el valor máximo del control sano (grupo 1). 


\section{DISCUSIÓN}

El daño del nervio lesionado por compresión genera cambios en la histología de dicha estructura; a través de esta experiencia se pudo confirmar que frente a una compresión disminuyó el número de NF. Este hallazgo parece lógico considerando que los neurofilamentos son elementos esenciales en el citoesqueleto neuronal, además de acuerdo con Hoffman et al. son un importante determinante del calibre axonal en las fibras mielinizadas. Por lo tanto, se demuestra que cuando ocurre una lesión nerviosa capaz de reducir el calibre del axón, la misma determina también disminución del número de NF.

El uso del US induce a una recuperación rápida del nervio tras su neurotomía. Se observa mayor número de fibras tipo A, lo que implica una actividad mayor de los Schwannocitos con precoz recuperación de las vainas de mielina (Crisci \& Ferreira). Akhlaghi et al. (2012) estudiaron la aplicación de US con 20 tipos de protocolo, con diferentes intensidades, frecuencias y modo de aplicación (pulsátil/continuo) y obtuvieron mejores resultados con aplicación de US $0,5 \mathrm{~W} / \mathrm{cm}^{2}, 1 \mathrm{MHz}$, modo pulsátil. Ellos afirmaron que los efectos no térmicos de los US son superiores a sus efectos térmicos y que la aplicación de la terapia en modo continuo podría producir efectos reversibles en la recuperación, ya que el modo continuo genera más calor. Farfán \& Matamala (2011) y Lowdon et al. determinaron que durante su reparación el nervio responde mejor frente a intensidades más altas en el rango de potencia de $0,5 \mathrm{~W} / \mathrm{cm}^{2}$ a $1 \mathrm{~W} /$ $\mathrm{cm}^{2}$, sin embargo Lowdon et al. enfatizaron que una radiación muy intensa provoca retraso en su recuperación.

En nuestro estudio, los resultados con el uso de US fueron significativos con la terapia de $1 \mathrm{~W} / \mathrm{cm}^{2}$ superando la reparación natural del grupo control lesionado, sin embargo la terapia de $0,5 \mathrm{~W} / \mathrm{cm} 2$ no logró diferenciarse del grupo control lesionado.

Estudios en animales han demostrado que tras una lesión nerviosa la aplicación de la irradiación por láser de baja intensidad (LBI) facilita la regeneración y produce aumento del calibre del nervio, capas de mielina más regulares (Mohammed et al., 2007) e incremento del número total de axones de grán calibre (Shamir et al.). Câmara et al. (2011) estudiaron los efectos histológicos del LBI (GaAs, 904 nm, $4 \mathrm{~J} / \mathrm{cm} 2$, $32 \mathrm{~s} /$ punto, 6 puntos, $26,3 \mathrm{~mW}$, spot $0,63 \mathrm{~cm}^{2}$ ) en la regeneración del nervio isquiático de ratones sometidos a axonotmesis. Estos autores observaron que en el grupo sometido a la irradiación por LBI hubo aumento del número total de axones y mejor calidad en el proceso de regeneración en comparación al grupo control, siendo la recupera- ción de los nervios más rápida y eficiente. Barbosa et al. (2010) compararon dos tipos de LBI: GaAlAs 660 nm (10 $\mathrm{J} / \mathrm{cm}^{2}, 30 \mathrm{~mW}$ y $0,06 \mathrm{~cm}^{2}, 20 \mathrm{~s}$, modo continuo) y de 830 $\mathrm{nm}\left(10 \mathrm{~J} / \mathrm{cm}^{2}, 30 \mathrm{~mW}\right.$ y $0,116 \mathrm{~cm}^{2}, 38,66 \mathrm{~s}$, modo continuo), en la regeneración del nervio isquiático tras compresión. Ellos encontraron recuperación funcional temprana utilizando el LBI de $660 \mathrm{~nm}$. Bagis et al. (2003) aplicaron

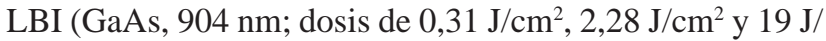
$\mathrm{cm}^{2}$; área del spot $0,28 \mathrm{~cm}^{2}$; modo pulsátil; 15 minutos, durante 6 días consecutivos) en el nervio isquiático de ratones sometidos a la compresión nerviosa y relataron que la terapia fue ineficaz en la reparación de la lesión, una vez que no hubo cambios electrofisiológicos y morfológicos en el nervio regenerado.

Con relación a los resultados antagónicos encontrados en las investigaciones estamos de acuerdo con Simunovic (1996) cuando señala que la efectividad clínica, o sea, el éxito o fracaso de la terapia por LBI depende de la aplicación de una dosis de irradiación correcta. La dosis de irradiación puede cambiar los procesos celulares, además, la aplicación de una dosis baja o muy baja puede producir ningún efecto o incluso efectos negativos o inhibidores (Hawkins \& Abrahamse, 2006). En nuestro estudio observamos que la dosis más alta de irradiación por LBI (10 J/ $\mathrm{cm}^{2}$ ) generó menor reparación nerviosa comparada a la irradiación por dosis más baja $\left(2 \mathrm{~J} / \mathrm{cm}^{2}\right)$, lo que confirma lo propuesto por Chen et al. (2005) que indican que la irradiación por LBI inadecuada podría impedir o retardar el proceso de regeneración del nervio, por lo tanto, señalamos que es primordial la utilización de un protocolo seguro para irradiación LBI en la práctica clínica.

Oliveira et al. (2012) evaluaron la eficacia de la terapia por LBI (AlGaAs, $830 \mathrm{~nm}, 40 \mathrm{~mW}$ ) y por US (KC 709M, ERA 3,5 cm², $1 \mathrm{MHz}, 0,4 \mathrm{~W} / \mathrm{cm}^{2}, 120 \mathrm{~s}$ ) en la regeneración del nervio isquiático tras compresión. Observaron que el LBI genera más benefícios en la recuperación biológica en comparación con el US. Nuestros resultados están de acuerdo a lo reportado por estos autores que señalan que a pesar que el LBI tiene efectividad superior en la regeneración del nervio isquiático lesionado, el US $\left(1 \mathrm{~W} / \mathrm{cm}^{2}\right)$ también presenta resultados significativamente mejores en la rehabilitación estructural que el grupo control lesionado.

En nuestra experiencia constatamos que el incremento en el número de NF se relacionó con la dosis aplicada de LBI y US. Observamos que la aplicación de una dosis más baja de LBI $\left(2 \mathrm{~J} / \mathrm{cm}^{2}\right)$ fue más eficaz que la dosis más alta $(10 \mathrm{~J} / \mathrm{cm} 2)$, sin embargo ambas aumentaron el número de 
NF. Con relación al US, la dosis de $1,0 \mathrm{~W} / \mathrm{cm}^{2}$ fue más eficaz que la dosis de $0,5 \mathrm{~W} / \mathrm{cm}^{2}$.

Concluimos que la aplicación de LBI y de US terapéutico aumenta el número de NF en nervios isquiáticos de rata sometidos a neuropraxia, siendo el LBI más eficaz en comparación al US. Además, la eficacia de las terapias para inducir la regeneración del nervio lesionado se relaciona con el tipo de protocolo utilizado, lo que demuestra la necesidad de establecer la adecuada dosis de irradiación con el propósito de obtener la mejor respuesta terapéutica, alcanzando así un tratamiento exitoso.

MATAMAla, F.; CORNEJO, R.; PAREDES, M.; FARFÁN, E.; GARRIDO, O. S. \& ALVES, N. Comparative analysis of the number of neurofilaments in rat sciatic nerve undergoing neuropraxia treated by low-level laser and therapeutic ultrasound. Int. $J$. Morphol., 32(1):369-374, 2014.

SUMMARY: Therapy by low-level laser (LLL) or ultrasound (US) are commonly used as treatment after nerve crush. The aim of this study was to determine the effectiveness of such treatments to repair the neuronal cytoskeleton evaluating the variation in the number of neurofilaments. For this an experimental design was performed, which involved 30 rats divided into 6 groups: 1 - control healthy; 2 - control injured; 3 - irradiated by LLL $2 \mathrm{~J} / \mathrm{cm} 2 ; 4$ - irradiated by LLL $10 \mathrm{~J} / \mathrm{cm} 2 ; 5$ - irradiated by US $0.5 \mathrm{~W} / \mathrm{cm} 2$ and 6 irradiated by US $1 \mathrm{~W} / \mathrm{cm} 2$. With the exception of group 1 all specimens were anesthetized and underwent right sciatic nerve compression using $40 \mathrm{~N}$ pressure for 45 seconds. Twenty-four hours after compression irradiation was started by LLL and US according protocol. In our research we found that the increase in the number of neurofilaments was related to the applied dose of LLL and US. The average value of neurofilaments / $0.25 \mathrm{~mm} 2$ obtained in each group was: 1 - 128; 2-100; 3-156; 4-140; 5-100; 6-148. We concluded that the application of LLL and therapeutic US increases the number of neurofilaments in rat sciatic nerve undergoing neuropraxia, with LLL being more effective compared to the US. Furthermore we concluded that the effectiveness of therapies to induce regeneration of injured nerve is related to the type of protocol used, demonstrating the need to establish an adequate radiation dose with the purpose of obtaining the best therapeutic response, thus achieving successful treatment.

\section{KEY WORDS: Sciatic nerve; Nerve crush; Neurofilaments; Low-level Laser Therapy; Ultrasound Therapy.}

\section{REFERENCIAS BIBLIOGRÁFICAS}

Akhlaghi, Z.; Mobarakeh, J. I.; Mokhtari, M.; Behnam, H.; Rahimi, A. A.; Khajeh Hosseini, M. S. \& Samiee, F. The effects of altered ultrasound parameters on the recovery of sciatic nerve injury. Iranian Biomed. J., 16(2):107-12, 2012.

Afifi, A. \& Bergman, R. A. Functional Neuroanatomy: text and atlas. 2nd ed. New York, McGraw-Hill, 2005.

Bagis, S.; Comelekoglu, U.; Coskun, B.; Milcan, A.; Buyukakilli, B.; Sahin, G.; Ozisik, S. \& Erdogan, C. No effect of GA-AS $(904 \mathrm{~nm})$ laser irradiation on the intact skin of the injured rat sciatic nerve. Lasers Med. Sci., 18(2):83-8, 2003.

Barbosa, R. I.; Marcolino, A. M.; de Jesus Guirro, R. R.; Mazzer, N.; Barbieri, C.H. \& de Cássia Registro Fonseca, M. Comparative effects of wavelengths of low-power laser in regeneration of sciatic nerve in rats following crushing lesion. Lasers Med. Sci., 25(3):423-30, 2010.

Câmara, C. N.; Brito, M. V.; Silveira, E. L.; Silva, D. S.; Simões, V. R. \& Pontes, R. W. Histological analysis of low-intensity laser therapy effects in peripheral nerve regeneration in Wistar rats. Acta Cir. Bras., 26(1):12-8, 2011.

Chen, Y. S.; Hsu, S. F.; Chiu, C. W.; Lin, J. G.; Chen, C. T. \& Yao, C. H. Effect of low-power pulsed laser on peripheral nerve regeneration in rats. Microsurgery, 25(1):83-9, 2005.
Crisci, A. R. \& Ferreira, A. L. Low-intensity pulsed ultrasound accelerates the regeneration of the sciatic nerve after neurotomy in rats. Ultrasound Med. Biol., 28(10):1335-41, 2002.

Ebenbichler, G. R.; Resch, K. L.; Nicolakis, P.; Wiesinger, G. F.; Uhl, F.; Ghanem, A H. \& Fialka, V. Ultrasound treatment for treating the carpal tunnel syndrome: randomized "sham" controlled trial. BMJ, 316(7133):731-5, 1998.

Farfán, E. \& Matamala, F. Tratamiento con ultrasonido continuo de alta intensidad en nervios isquiáticos de rata (Sprague Dawley) lesionados por compresión. Int. J. Morphol., 29(3):787-91, 2011.

Hawkins, D. H. \& Abrahamse, H. The role of laser fluence in cell viability, proliferation, and membrane integrity of wounded human skin fibroblasts following helium-neon laser irradiation. Lasers Surg. Med., 38(1):74-83, 2006.

Hoffman, P. N.; Griffin, J. W. \& Price, D. L. Control of axonal caliber by neurofilament transport. J. Cell Biol., 99(2):70514, 1984.

Hursh, J. B. Conduction velocity and diameter of nerve fibers. Am. J. Physiol., 127:131-9, 1939.

Jaquet, J. B.; Luijsterburg, A. J.; Kalmijn, S.; Kuypers, P. D.; 
MATAMALA, F.; CORNEJO, R.; PAREDES, M.; FARFÁN, E.; GARRIDO, O. S. \& ALVES, N. Análisis comparativo del número de neurofilamentos en nervios isquiáticos de rata sometidos a neuropraxia tratadas con láser de baja intensidad y ultrasonido terapéutico. Int. J. Morphol., 32(1):369-374, 2014

Hofman, A. \& Hovius, S. E. Median, ulnar, and combined median-ulnar nerve injuries: functional outcome and return to productivity. J. Trauma, 51(4):687-92, 2001.

Lowdon, I. M.; Seaber, A. V. \& Urbaniak, J. R. An improved method of recording rat tracks for measurement of the sciatic functional index of de Medinaceli. J. Neurosci. Methods, 24(3):279-81, 1988.

Martínez Morillo, M.; Pastor Vega, J. M. \& Sendra Portero, F. Manual de Medicina Física. Madrid, Harcourt Brace, 1998. p.448.

Miloro, M. \& Repasky, M. Low-level laser effect on neurosensory recovery after sagittal ramus osteotomy. Oral Surg. Oral Med. Oral Pathol. Oral Radiol. Endod., 89(1):12-8, 2000.

Mohammed, I. F.; Al-Mustawfi, N. \& Kaka, L. N. Promotion of regenerative processes in injured peripheral nerve induced by low-level laser therapy. Photomed. Laser Surg., 25(2):107-11, 2007.

Oliveira, F. B.; Pereira, V. M. D.; Trindade, A. P. N. T.; Shimano, A. C.; Gabriel, R. E. C. D. \& Borges, A. P. O. Action of therapeutic laser and ultrasound in peripheral nerve regeneration. Acta Ortop. Bras., 20(2):98-103, 2012.

Rochkind, S. \& Ouaknine, G. E. New trend in neuroscience: lowpower laser effect on peripheral and central nervous system (basic science, preclinical and clinical studies). Neurol. Res., 14(1):2-11, 1992.

Seddon, I. \& Herbert, J. Surgical disorders of the peripheral nerves. London, Churchill Livingstone, 1975.

Shamir, M. H.; Rochkind, S.; Sandbank, J. \& Alon, M. Doubleblind randomized study evaluating regeneration of the rat transected sciatic nerve after suturing and postoperative lowpower laser treatment. J. Reconstr. Microsurg., 17(2):133-8, 2001.

Simunovic, Z. Low level laser therapy with trigger points technique: a clinical study on 243 patients. J. Clin. Laser Med. Surg., 14(4):163-7, 1996.

Snyder-Mackler, L. \& Collender, S. L. Therapeutic uses of light in rehabilitation. In: Michlovitz, S. L. Thermal Agents in Rehabilitation. Contemporary Perspectives in Rehabilitation. 3rd ed. Philadelphia, F.A. Davis, 1996.

Solé, P.; Moller, A. \& Reininger, D. Revisión bibliográfica del uso de láser de baja potencia como tratamiento en alteraciones del nervio alveolar inferior en osteotomía sagital de rama. Int. J. Odontostomat., 6(3):307-11, 2012.

\author{
Dirección para correspondencia \\ Fernando Matamala \\ Universidad de La Frontera \\ Casilla 54-D \\ Temuco \\ CHILE
}

Email: matamala.fernando@gmail.com

Received: 13-08-2013

Accepted: 09-01-2014 